\title{
THE $\Gamma$-FUNCTION REVISITED: POWER SERIES EXPANSIONS AND REAL-IMAGINARY ZERO LINES
}

\author{
JAN BOHMAN AND CARL-ERIK FRÖBERG
}

\begin{abstract}
Explicit power series expansions of the gamma function are given close to $-10,-2,-1,0,1,2,3,4$, and 10 together with formulas that can be used in other integer points. Further, curves along which the real or imaginary part of the function vanish are presented.
\end{abstract}

\section{INTRODUCTION}

When just one isolated value of the $\Gamma$-function is needed, it may be computed in a straightforward fashion by Stirling's formula, followed by repeated use of the functional relation $\Gamma(z+1)=z \Gamma(z)$. However, if more detailed knowledge of the function is desired within a small area close to the real axis, a different approach might be reasonable. For $n=2,3,4,10$ we give expansions of the form

$$
\Gamma(n+1+z)=n !\left(1+d_{1} z+d_{2} z^{2}+\cdots\right),
$$

and for $n=0,1,2,10$,

$$
\begin{aligned}
(-1)^{n} n ! \Gamma(-n+z)= & n /(1-z)-1 /((n+1)(1+z)) \\
& +z^{-1}\left(1+f_{1} z+f_{2} z^{2}+\cdots\right) .
\end{aligned}
$$

Reasonably fast convergence is obtained, at least for $|z|<1$.

\section{A USEFUL ALGORITHM}

We will propose an algorithm for computation of certain finite products (also infinite when convergent). This will give us a tool for determining various sets of coefficients. Let

$$
P=\prod_{k=1}^{n}\left(1+\frac{x}{p_{k}}\right)^{m_{k}}=1+u_{1} x+u_{2} x^{2}+\cdots,
$$

where $p_{k}$ and $m_{k}$ are given real numbers $\neq 0$ and the coefficients $u_{k}$ are to be determined. Taking logarithms and differentiating, we obtain

$$
\ln P=\sum_{k=1}^{n} m_{k} \ln \left(1+\frac{x}{p_{k}}\right), \quad \frac{P^{\prime}}{P}=\sum_{k=1}^{n} \frac{m_{k}}{x+p_{k}} .
$$

Received October 10, 1990; revised February 19, 1991.

1991 Mathematics Subject Classification. Primary 33B15, 65D20. 
Now we define

$$
f_{r}(x)=(-1)^{r+1} \sum_{k=1}^{n} m_{k}\left(x+p_{k}\right)^{-r}, \quad r=1,2,3, \ldots,
$$

with the obvious property

$$
f_{r}^{\prime}(x)=r f_{r+1}(x)
$$

Further, we put

$$
f_{r}(0)=w_{r}=(-1)^{r+1} \sum_{k=1}^{n} m_{k} p_{k}^{-r} .
$$

Repeated differentiation gives

$$
\begin{aligned}
P^{\prime} & =P f_{1}, \\
P^{\prime \prime} & =P^{\prime} f_{1}+P f_{2}, \\
P^{\prime \prime \prime} & =P^{\prime \prime} f_{1}+2 P^{\prime} f_{2}+2 P f_{3}, \\
P^{\text {iv }} & =P^{\prime \prime \prime} f_{1}+3 P^{\prime \prime} f_{2}+6 P^{\prime} f_{3}+6 P f_{4},
\end{aligned}
$$

Setting $x=0$ and observing that $P^{(r)}(0)=r ! u_{r}$ with $P(0)=1$, we get the system

$$
\begin{aligned}
u_{1} & =w_{1} \\
2 u_{2} & =w_{1} u_{1}+w_{2} \\
3 u_{3} & =w_{1} u_{2}+w_{2} u_{1}+w_{3} \\
4 u_{4} & =w_{1} u_{3}+w_{2} u_{2}+w_{3} u_{1}+w_{4}
\end{aligned}
$$

Note that if the $m_{k}$ are positive integers, then $P$ is a polynomial, and all $u_{k}$ with $k>\sum_{r=1}^{n} m_{r}$ are zero. Using Cramer's rule, we find after some manipulation:

$$
u_{n}=\frac{1}{n !}\left|\begin{array}{ccccc}
w_{1} & -1 & 0 & \ldots & 0 \\
w_{2} & w_{1} & -2 & \ldots & 0 \\
w_{3} & w_{2} & w_{1} & \ldots & \ldots \\
\cdots & \cdots & \cdots & \cdots & -(n-1) \\
w_{n} & w_{n-1} & \cdots & \cdots & w_{1}
\end{array}\right|
$$

In practical computation the system (5) should be solved recursively. However, we also present the first few values explicitly:

$$
\begin{aligned}
& u_{1}=w_{1}, \\
& u_{2}=\frac{1}{2}\left(w_{1}^{2}+w_{2}\right), \\
& u_{3}=\frac{1}{6}\left(w_{1}^{3}+3 w_{1} w_{2}+2 w_{3}\right), \\
& u_{4}=\frac{1}{24}\left(w_{1}^{4}+6 w_{1}^{2} w_{2}+8 w_{1} w_{3}+3 w_{2}^{2}+6 w_{4}\right),
\end{aligned}
$$

It is interesting to observe that these expressions also appear in a quite different context. Consider the permutations of, e.g., four objects, which we denote 
$1,2,3$, and 4. Let $(1)(2)(3)(4)$ be the identical permutation; (13)(24) the permutation where 1 and 3 switch places, and the same for 2 and 4; (3)(142) the permutation where 3 keeps its place, while 1 goes over into 4,4 into 2 , and 2 into 1; etc. Denote a part permutation involving $k$ objects by $s_{k}$; then, e.g., (1)(3)(24) is written $s_{1}^{2} s_{2},(2)(143)$ as $s_{1} s_{3}$, and so on. Writing down all $24=4$ ! permutations and dividing by 24 , we get the so-called cycle index

$$
S_{4}=\frac{1}{24}\left(s_{1}^{4}+6 s_{1}^{2} s_{2}+8 s_{1} s_{3}+3 s_{2}^{2}+6 s_{4}\right),
$$

an expression with exactly the same structure as $u_{4}$ in (7).

3. POWER SERIES EXPANSION FOR $\Gamma(z+2)$

As is well known, the $\Gamma$-function is defined in product form by

$$
\Gamma(z+1)=e^{-\gamma z} \prod_{n=1}^{\infty} \frac{e^{z / n}}{1+z / n},
$$

where as usual $\gamma$ is the Euler constant, $\gamma=0.5772156649 \ldots$. Taking logarithms, we obtain

$$
\begin{aligned}
\ln \Gamma(z+1) & =-\gamma z+\sum_{n=1}^{\infty}\left[\frac{z}{n}-\ln \left(1+\frac{z}{n}\right)\right] \\
& =(1-\gamma) z-\ln (1+z)+\sum_{n=2}^{\infty} \frac{(-1)^{n}(\zeta(n)-1) z^{n}}{n},
\end{aligned}
$$

where $\zeta(n)=\sum_{k=1}^{\infty} k^{-n}, n>1$, is the Riemann zeta function. Now we introduce the notations

$$
\left\{\begin{array}{l}
g_{1}=1-\gamma, \\
g_{r}=(-1)^{r}(\zeta(r)-1), \quad r=2,3,4, \ldots,
\end{array}\right.
$$

and obtain $\Gamma(z+2)=\exp \left(g_{1} z+\frac{1}{2} g_{2} z^{2}+\frac{1}{3} g_{3} z^{3}+\cdots\right)$. We can then construct the corresponding power series

$$
\begin{aligned}
G(z) & =\Gamma(z+2)=\exp \left(g_{1} z+\frac{1}{2} g_{2} z^{2}+\cdots\right) \\
& =1+c_{1} z+c_{2} z^{2}+c_{3} z^{3}+\cdots .
\end{aligned}
$$

Differentiation gives

$$
G^{\prime}(z)=G(z) \cdot\left(g_{1}+g_{2} z+g_{3} z^{2}+\cdots\right)=c_{1}+2 c_{2} z+3 c_{3} z^{2}+\cdots .
$$

Hence, we obtain a linear system

$$
\begin{aligned}
c_{1} & =g_{1}, \\
2 c_{2} & =g_{1} c_{1}+g_{2}, \\
3 c_{3} & =g_{1} c_{2}+g_{2} c_{1}+g_{3},
\end{aligned}
$$

which has the same structure as (5). The solution is

$$
c_{n}=\frac{1}{n !}\left|\begin{array}{cccccc}
g_{1} & -1 & 0 & 0 & \cdots & 0 \\
g_{2} & g_{1} & -2 & 0 & \cdots & 0 \\
g_{3} & g_{2} & g_{1} & -3 & \cdots & 0 \\
\cdots & \cdots & \cdots & \cdots & \cdots & \cdots \\
g_{n} & g_{n-1} & \cdots & \cdots & \cdots & g_{1}
\end{array}\right| \text {. }
$$


TABLE 1

Numerical values of $g_{n}$ (9) and $c_{n}$ (11). For larger values of $k$, $(-1)^{k}\left(2^{-(k+1)}-3^{-(k+1)}\right)$ is a remarkably good approximation of $c_{k}$.

\begin{tabular}{rrrcrr}
$n$ & \multicolumn{1}{c}{$c_{n}$} & $n$ & \multicolumn{1}{c}{$g_{n}$} & \multicolumn{1}{c}{$c_{n}$} \\
1 & $0.4227843351+0$ & $0.4227843351+0$ & 11 & $-0.4941886041-3$ & $-0.2409414358-3$ \\
2 & $0.6449340668+0$ & $0.4118403304+0$ & 12 & $0.2460865533-3$ & $0.1216738065-3$ \\
3 & $-0.2020569032+0$ & $0.8157691925-1$ & 13 & $-0.1227133476-3$ & $-0.6079289132-4$ \\
4 & $0.8232323371-1$ & $0.7424901075-1$ & 14 & $0.6124813506-4$ & $0.3045355703-4$ \\
5 & $-0.3692775514-1$ & $-0.2669820687-3$ & 15 & $-0.3058823631-4$ & $-0.1523493590-4$ \\
& & & & & \\
6 & $0.1734306198-1$ & $0.1115404572-1$ & 16 & $0.1528225941-4$ & $0.7621779696-5$ \\
7 & $-0.8349277382-2$ & $-0.2852645821-2$ & 17 & $-0.7637197638-5$ & $-0.3812110400-5$ \\
8 & $0.4077356198-2$ & $0.2103933341-2$ & 18 & $0.3817293265-5$ & $0.1906491658-5$ \\
9 & $-0.2008392826-2$ & $-0.9195738388-3$ & 19 & $-0.1908212717-5$ & $-0.9533877803-6$ \\
10 & $0.9945751278-3$ & $0.4903884508-3$ & 20 & $0.9539620339-6$ & $0.4767416946-6$
\end{tabular}

The coefficients $c_{n}$ are well known; they were given by Davis [1, p. 186] and were denoted there by $E_{n}$. On the other hand, only very few power series expansions are reported in the Handbook of Mathematical Functions [2]; the most interesting is one for $1 / \Gamma(z)$. We note here that $z= \pm 1$ leads to the relation $c_{1}+c_{3}+c_{5}+\cdots=c_{2}+c_{4}+c_{6}+\cdots=\frac{1}{2}$.

The numerical values of $c_{n}$ are obtained recursively from the linear system. They are displayed in Table 1 together with the constants $g_{n}$.

\section{POWER SERIES EXPANSIONS FOR $\Gamma(n+1+z)$}

Using the fundamental relation $\Gamma(z+1)=z \Gamma(z)$ repeatedly, we find, when $n \geq 2$,

$$
\Gamma(n+1+z)=(z+n)(z+n-1) \cdots(z+2) \Gamma(z+2)
$$

or, after division by $\Gamma(n+1)=n !$,

$$
\frac{\Gamma(n+1+z)}{\Gamma(n+1)}=\left(1+\frac{z}{2}\right)\left(1+\frac{z}{3}\right) \cdots\left(1+\frac{z}{n}\right)\left(1+c_{1} z+c_{2} z^{2}+\cdots\right) .
$$

The product $\left(1+\frac{z}{2}\right)\left(1+\frac{z}{3}\right) \cdots\left(1+\frac{z}{n}\right)=1+a_{1} z+\cdots+a_{n-1} z^{n-1}$ is computed as in (5) with $w_{r}=(-1)^{r+1} \sum_{k=2}^{n} k^{-r}$ (note that $w_{r}$ depends on $n$ ). Finally, we compute the product

$$
\left(1+a_{1} z+\cdots+a_{n-1} z^{n-1}\right)\left(1+c_{1} z+c_{2} z^{2}+\cdots\right)=1+d_{1} z+d_{2} z^{2}+\cdots
$$

and obtain the result

$$
d_{k}=\sum_{r=0}^{k} a_{r} c_{k-r}
$$

with $a_{0}=c_{0}=1$ and $a_{k}=0$ when $k \geq n$. It is obvious that also the coefficients $d_{1}, d_{2}, d_{3}, \ldots$ depend on $n$. The case $n=1$ is trivial, since then $d_{k}=c_{k}$. Also the case $n=0$ is easy to handle, since the relation $\Gamma(z+1)=$ $\Gamma(z+2) /(z+1)$ leads to $d_{k}=c_{k}-d_{k-1}$ with $d_{0}=1$. For $n=2,3,4$ and 10 , numerical values of $d_{k}$ are given in Table 2 . 
TABLE 2

The coefficients $d_{k}$ for computation of $\Gamma(n+1+z)=\Gamma(n+1) \cdot$ $\left(1+d_{1} z+d_{2} z^{2}+\cdots\right)$. When $n=1$ we have $d_{k}=c_{k}$ (see Table 1). When $n=0$, use $\Gamma(1+z)=\left(1+c_{1} z+c_{2} z^{2}+\cdots\right) /(1+z)$.

$\begin{array}{rrccc}k & n=2 & n=3 & n=4 & n=10 \\ 1 & 0.9227843351+00 & 0.1256117668+01 & 0.1506117668+01 & 0.2351752589+01 \\ 2 & 0.6232324980+00 & 0.9308272763+00 & 0.1244856693+01 & 0.2812953288+01 \\ 3 & 0.2874970845+00 & 0.4952412505+00 & 0.7279480695+00 & 0.2278217646+01 \\ 4 & 0.1150374704+00 & 0.2108698319+00 & 0.3346801445+00 & 0.1403786967+01 \\ 5 & 0.3685752331-01 & 0.7520334677-01 & 0.1279208047+00 & 0.7012189188+00 \\ & & & & \\ 6 & 0.1102055468-01 & 0.2330639579-01 & 0.4210723248-01 & 0.2955229663+00 \\ 7 & 0.2724377038-02 & 0.6397895266-02 & 0.1222449421-01 & 0.1079966380+00 \\ 8 & 0.6776104301-03 & 0.1585736109-02 & 0.3185209926-02 & 0.3491152784-01 \\ 9 & 0.1323928315-03 & 0.3582629749-03 & 0.7546970023-03 & 0.1013547328-01 \\ 10 & 0.3060153141-04 & 0.7473247525-04 & 0.1642982190-03 & 0.2674217142-02 \\ & & & & \\ 11 & 0.4252789587-05 & 0.1445330006-04 & 0.3313641887-04 & 0.6474128057-03 \\ 12 & 0.1203088620-05 & 0.2620685149-05 & 0.6234010164-05 & 0.1449479266-03 \\ 13 & 0.4401195495-07 & 0.4450414950-06 & 0.1100212782-05 & 0.3020944116-04 \\ 14 & 0.5711138227-07 & 0.7178203392-07 & 0.1830424077-06 & 0.5893770496-05 \\ 15 & -0.8157376050-08 & 0.1087975137-07 & 0.2882525985-07 & 0.1081535153-05 \\ & & & & \\ 16 & 0.4311748695-08 & 0.1592623345-08 & 0.4312561188-08 & 0.1874504774-06 \\ 17 & -0.1220552013-08 & 0.2166975524-09 & 0.6148533886-09 & 0.3079697095-07 \\ 18 & 0.4364575319-09 & 0.2960686106-10 & 0.8378124916-10 & 0.4811678892-08 \\ 19 & -0.1419514757-09 & 0.3534368324-11 & 0.1093608359-10 & 0.7169506577-09 \\ 20 & 0.4780444902-10 & 0.4872904734-12 & 0.1370882554-11 & 0.1021391242-09\end{array}$

\section{POWER SERIES EXPANSION RELATED to $\Gamma(-n+z)$}

By straightforward calculation we find

$$
\Gamma(z-n)=\frac{(-1)^{n} \Gamma(z+2)}{n ! z}\left\{(1+z)(1-z)\left(1-\frac{z}{2}\right) \cdots\left(1-\frac{z}{n}\right)\right\}^{-1} .
$$

Hence, $m_{k}=-1$ and $p_{k}=-k$, if we add an extra value $p_{0}=1$. In this way we obtain

$$
w_{r}=(-1)^{r}+\sum_{k=1}^{n} k^{-r}
$$

The coefficients $b_{r}$ for the reciprocal of the expression within braces are again obtained from (5). The final coefficients $e_{r}$ defined through

$$
\Gamma(z-n)=\left((-1)^{n} / n ! z\right)\left(1+e_{1} z+e_{2} z^{2}+\cdots\right)
$$

can be computed as in (14). When $n=0$, we have

$$
\begin{aligned}
\Gamma(z) & =\frac{\Gamma(z+2)}{z(z+1)}=\frac{1}{z}\left(1+c_{1} z+c_{2} z^{2}+\cdots\right)\left(1-z+z^{2}-\cdots\right) \\
& =\frac{1}{z}\left(1+e_{1} z+e_{2} z^{2}+\cdots\right),
\end{aligned}
$$

and hence $e_{r}=c_{r}-e_{r-1}$ with $e_{0}=1$. Obviously, the coefficients $e$ and $d$ are identical when $n=0$. 


\section{TABLE 3}

Coefficients $f_{k}$ for $n=0,1,2,10$ and $k=1(1) 20$ defined by $(-1)^{n} n ! \Gamma(-n+z)=n /(1-z)-1 /(n+1)(1+z)+$ $\frac{1}{z}\left(1+f_{1} z+f_{2} z^{2}+\cdots\right)$.

$\begin{array}{rrrcc}k & n=0 & n=1 & n=2 & n=10 \\ 1 & 0.4227843351+0 & -0.7721566490-1 & -0.7438823316+0 & -0.7557338320+1 \\ 2 & -0.1094400467-1 & -0.8815966957-1 & -0.4601008354+0 & -0.5728188072+1 \\ 3 & 0.9252092392-1 & 0.4361254346-2 & -0.2256891633+0 & -0.3986202989+1 \\ 4 & -0.1827191317-1 & -0.1391065882-1 & -0.1267552405+0 & -0.2585789587+1 \\ 5 & 0.1800493110-1 & 0.4094272277-2 & -0.5928334797-1 & -0.1580626439+1 \\ & & & & \\ 6 & -0.6850885379-2 & -0.2756613102-2 & -0.3239828709-1 & -0.9220023064+0 \\ 7 & 0.3998239558-2 & 0.1241626456-2 & -0.1495751709-1 & -0.5176270823+0 \\ 8 & -0.1894306217-2 & -0.6526797612-3 & -0.8131438305-2 & -0.2822577798+0 \\ 9 & 0.9747323780-3 & 0.3220526168-3 & -0.3743666536-2 & -0.1503697153+0 \\ 10 & -0.4843439272-3 & -0.1622913104-3 & -0.2034124578-2 & -0.7875712620-1 \\ & & & & \\ 11 & 0.2434024914-3 & 0.8111118100-4 & -0.9359511081-3 & -0.4069995193-1 \\ 12 & -0.1217286849-3 & -0.4061750386-4 & -0.5085930579-3 & -0.2083900601-1 \\ 13 & 0.6093579356-4 & 0.2031828969-4 & -0.2339782393-3 & -0.1059168640-1 \\ 14 & -0.3048223652-4 & -0.1016394683-4 & -0.1271530665-3 & -0.5358239971-2 \\ 15 & 0.1524730062-4 & 0.5083353797-5 & -0.5849317943-4 & -0.2700107474-2 \\ & & & & \\ 16 & -0.7625520927-5 & -0.2542167130-5 & -0.31788756884-4 & -0.1357696113-2 \\ 17 & 0.3813410527-5 & 0.1271243397-5 & -0.1462313503-4 & -0.6812730636-3 \\ 18 & -0.1906918869-5 & -0.6356754719-6 & -0.7947242984-5 & -0.3415598177-3 \\ 19 & 0.9535310888-6 & 0.3178556169-6 & -0.3655765875-5 & -0.1710444012-3 \\ 20 & -0.4767893943-6 & -0.1589337773-6 & -0.1986816715-5 & -0.8563572098-4\end{array}$

It turned out that the coefficients $e_{k}$ for large values of $k$ essentially behave as $n+(-1)^{k} /(n+1)$. For this reason, we tabulate in Table 3 other coefficients $f_{k}$ defined by

$$
f_{k}=e_{k}-\left(n+(-1)^{k} /(n+1)\right),
$$

giving much better accuracy. If a complete power series is wanted, it is an easy matter to recompute the coefficients $e_{k}$. Using the new coefficients, we find

$$
\Gamma(-n+z)=\frac{(-1)^{n}}{(n-1) !(1-z)}-\frac{(-1)^{n}}{(n+1) !(1+z)}+\frac{(-1)^{n}}{n ! z}\left(1+f_{1} z+f_{2} z^{2}+\cdots\right)
$$

This formula will give reasonable results, even when $|z|$ is only slightly less than 1 , and when $|z|<\frac{1}{2}$, we will get 10-digit accuracy. In the case $n=0$ we get

$$
f_{k}=d_{k}+(-1)^{k-1}=e_{k}+(-1)^{k-1}, \quad k=1,2,3, \ldots,
$$

with

$$
\Gamma(z)=-\frac{1}{1+z}+\frac{1}{z}\left(1+f_{1} z+f_{2} z^{2}+\cdots\right)
$$

and, of course, $\Gamma(1+z)=z \Gamma(z)$. For larger values of $k$, the coefficients $f_{k}$ behave approximately as $-2^{-k}\left(n(n-1)+(-1)^{k} /(n+1)(n+2)\right)$.

Since the coefficients vary in a very regular manner for larger values of $k$, at least 8-digit accuracy is easily attainable when $|z| \leq 1$. 


\section{ZERO LINES FOR THE REAL AND THE IMAGINARY PART}

As is well known, the $\Gamma$-function has no zeros. However, the function values are real along the real axis, and we now ask if there are curves in the complex plane where the real or the imaginary part vanishes. It will then be helpful to consider points $z=x+\varepsilon e^{i v}$ with $x$ real. We start with the imaginary part and find

$$
\operatorname{Im} \Gamma(z)=\varepsilon \Gamma^{\prime}(x) \sin v+\frac{1}{2} \varepsilon^{2} \Gamma^{\prime \prime}(x) \sin 2 v+O\left(\varepsilon^{3}\right) .
$$

This part will vanish if $\Gamma^{\prime}(x)=0$, and further we see that we must have $\sin 2 v=0$, giving $v=\pi / 2$. The points defined by $\Gamma^{\prime}(x)=0$ are well known and (except for the first one) lie between the poles. A few of them are given in [2] $(1.46163,-0.504,-1.573,-2.611,-3.635,-4.658,-5.667,-6.678$, etc.).

Since the real part cannot vanish in a regular point on the real axis, we have to investigate the situation close to the poles. We have

$$
\Gamma(z-n)=\frac{(-1)^{n}}{n ! z}\left(1+e_{1} z+e_{2} z^{2}+\cdots\right)
$$

and define

$$
R=\Gamma\left(-n+\varepsilon e^{i v}\right)=\frac{(-1)^{n}}{n !}\left(\varepsilon^{-1} e^{-i v}+e_{1}+e_{2} \varepsilon e^{i v}+\cdots\right)
$$

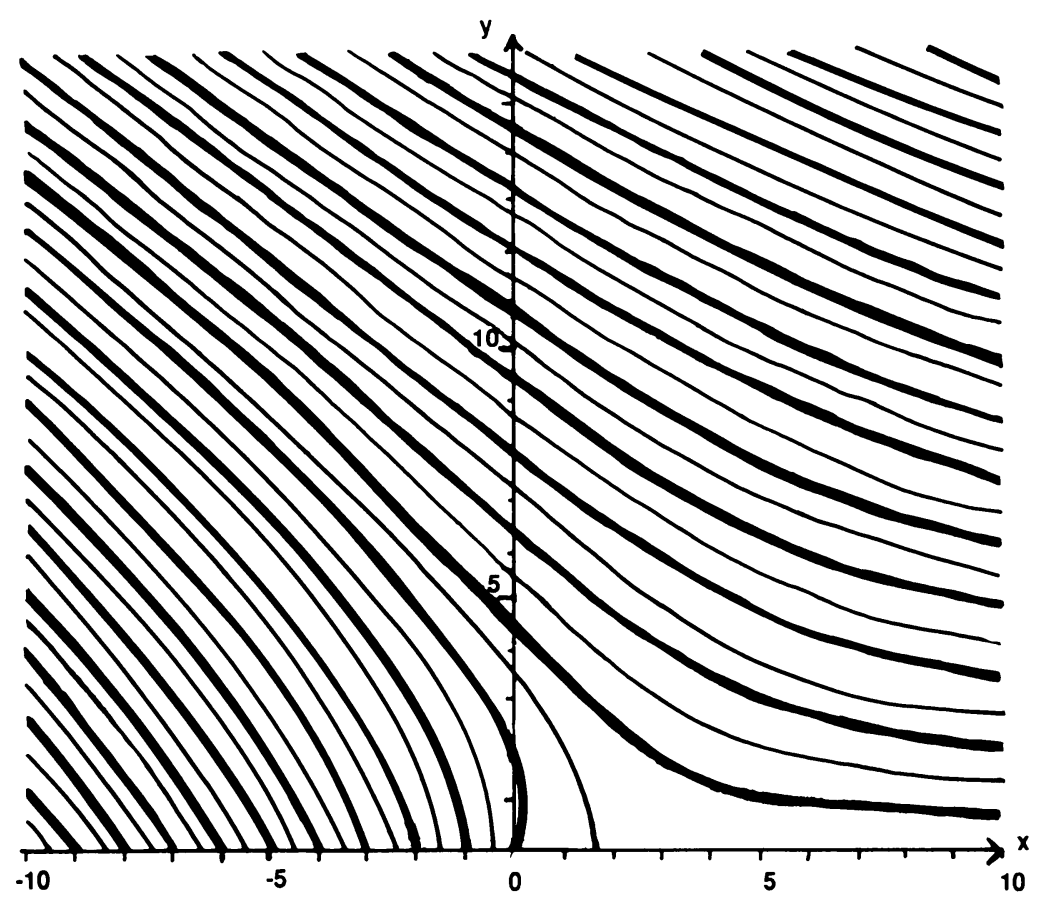

$\operatorname{Im} \Gamma(z)=0 ; \operatorname{Re} \Gamma(\mathbf{z})=0$ 
The real part is

$$
\operatorname{Re}(R)=\frac{(-1)^{n}}{n !}\left[\varepsilon^{-1} \cos v+e_{1}+O(\varepsilon)\right]=0
$$

with $e_{1}=f_{1}+n-1 /(n+1), n=0,1,2, \ldots$, which gives $\cos v=-e_{1} \varepsilon$, i.e., $v \simeq \pi / 2+e_{1} \varepsilon$. Hence, both types of curves pass vertically over the real axis (note, however, that this vertical direction changes very rapidly owing to the large values of $e_{1}$ ). We see that there is one curve with vanishing real part passing through every pole.

The practical computation is tedious but straightforward, and the resulting figure is only intended to give a general impression of the family of curves under discussion.

\section{BIBLIOGRAPHY}

1. H. T. Davis, Tables of the higher mathematical functions, vol. 1, Principia Press, Bloomington, IN, 1933.

2. M. Abramowitz and I. A. Stegun, Handbook of mathematical functions, National Bureau of Standards Appl. Math. Ser., no. 55, 1964.

3. E. R. Hansen, A table of series and products, Prentice-Hall, Englewood Cliffs, NJ, 1978.

Department of Computer Science, Lund University, Box 118, S-221 00 Lund, Sweden 